\title{
Soziale Gerechtigkeit im Spiegel der Einkommensverteilung in Deutschland
}

Im Zuge der Klagen über einen „ausufernden“ Sozialstaat wird häufig gefordert, das Ziel sozialer Gerechtigkeit auf die Gewährleistung von formaler Chancengleichheit zu begrenzen. Eine systematische Erörterung der aus dem Sozialstaatsprinzip ableitbaren Ziele der Chancen-, Leistungs- und Bedarfsgerechtigkeit offenbart jedoch vielfältige Interdependenzen. Letztlich ist die Linderung von Armut und Einkommensunsicherheit eine wesentliche Voraussetzung für den Erfolg sozialinvestiver Strategien. Da jedoch anhand der Entwicklung der Einkommensverteilung erhebliche Defizite insbesondere bei der Umsetzung von Chancen- und Bedarfsgerechtigkeit erkennbar sind, ist eine Umverteilung über steuerfinanzierte Transfers nach wie vor erforderlich.

\section{Soziale Gerechtigkeit - ein interdependentes Zielsystem}

Mit dem von Amartya Sen (1999) entwickelten Konzept der „capabilities“ - üblicherweise übersetzt mit „Verwirklichungschancen"-ist ein Begriffssystem eingeführt worden, das den Terminus der sozialen Gerechtigkeit zumindest vorübergehend in den Hintergrund gedrängt hat. Dies ist insofern erstaunlich, als der theoretische Ansatz von Sen kein Gegenentwurf zu den Inhalten sozialer Gerechtigkeit ist. Es handelt sich vielmehr um einen Erklärungsansatz, der von dem gleichen Recht aller Menschen, ihr Leben selbstbestimmt - also autonom - führen zu können, ausgeht und auf das Zusammenwirken verschiedener gesellschaftlicher Ebenen, individueller Veranlagungen und Präferenzen eingeht. Der Ausgangspunkt entspricht einem Ziel sozialer Gerechtigkeit, das sozialphilosophisch begründet werden kann (vgl. dazu Kronauer/Schmid in diesem Heft). Das daran anknüpfende analytische Konzept impliziert Forschungsaufgaben wie auch Handlungsempfehlungen an Gesellschaft und Politik. Fragen nach einzelnen Aspekten sozialer Gerechtigkeit sind damit aber keineswegs überflüssig; ihnen soll mit dem folgenden Beitrag zur Einkommensverteilung in Deutschland nachgegangen werden. Dabei wird eine dezisionistische Perspektive eingenommen: Maßgeblich sind die Willensbekundungen der Bürgerinnen und Bürger, wie sie sich in einer repräsentativen Demokratie im gesetzlichen Rahmen niederschlagen.

Der Sozialrechtler Zacher (1977, S. 154) charakterisiert den Sozialstaat als einen
Staat, „der den wirtschaftlichen und wirtschaftlich bedingten Verhältnissen auch in der Gesellschaft wertend, sichernd und verändernd mit dem Ziel gegenübersteht, jedermann ein menschenwürdiges Dasein zu gewährleisten, Wohlstandsunterschiede zu verringern und Abhängigkeitsverhältnisse zu beseitigen oder zu kontrollieren“. In entsprechenden Gesetzen, Richtlinien, Verordnungen und verfassungsgerichtlichen Entscheidungen haben sich Chancengleichheit, Leistungsgerechtigkeit und Bedarfsgerechtigkeit als dominierende Ausprägungen sozialer Gerechtigkeit herauskristallisiert. Dies spiegelt sich in der Sozialordnung, die insbesondere die Arbeitsmarktordnung, Mitbestimmungsregelungen, das System der sozialen Sicherung und Elemente des Steuersystems umfasst. Die in neuerer Zeit besonders hervorgehobenen Ziele der Gender- und der Generationengerechtigkeit lassen sich vor diesem Hintergrund als Querschnittsaufgaben interpretieren, da es bei Forderungen nach Gerechtigkeit zwischen den Geschlechtern und zwischen Generationen letztlich wiederum um gleiche Chancen, gerechte Leistungsbewertung und den Ausgleich von Bedarfsdeckungsmöglichkeiten geht. Generationengerechtigkeit ist allerdings nicht auf die Beziehungen zwischen den zu einem Zeitpunkt zusammenlebenden Altersgruppen beschränkt. Daneben sind auch intertemporale Aspekte zur Wahrung einer „Längsschnittgerechtigkeit" zu berücksichtigen, die auf die Höhe des "Generationenerbes“ im Sinne eines lebenszeitbezogenen Transfersaldos gerichtet sind.

Die analytische Zerlegung des Gerechtigkeitsziels sollte allerdings nicht als Eigenständigkeit der verschiedenen Aspekte missverstanden werden. Denn zwischen allen genannten Teilzielen bestehen vielschichtige Interdependenzen. Dies ist in Abbildung 1 skizziert. Die weitgehende Verwirklichung der Chancengleichheit insbesondere im Bildungssystem und auf dem Arbeitsmarkt - stellt eine wesentliche Voraussetzung für die Anerkennung der sich über den Markt ergebenden Einkommens- und Vermögensverteilung als „leistungsgerecht" und damit für die Akzeptanz der resultierenden Ungleichheit dar. Auch die Zieldimension der Bedarfsgerechtigkeit steht gegenüber der einer Chancengleichheit in komplementärer Beziehung; denn Verletzungen der Bedarfsgerechtigkeit, insbesondere Armut und Ausgrenzung, verstärken die Ungleichheiten der Chancen. Damit sind indirekt auch Bedarfs- und Leistungsgerechtigkeit komplementär. Andererseits ist zwischen Leistungs- und Bedarfsgerechtigkeit insofern ein Zielkonflikt angelegt, als bei ausgeprägtem Leistungsprinzip und hoher Ungleichheit der Markteinkommen mit Verletzungen des Ziels der Bedarfsgerechtigkeit zu rechnen ist, sofern keine ausgleichenden staatlichen Transferzahlungen erfolgen und die Auswirkungen des Leistungsprinzips korrigieren. Diese konträre Beziehung ist wiederum insoweit übergreifend, als Bedarfsgerechtigkeit eine Voraussetzung für die Annäherung der Chancen ist und damit indirekt auch zwischen Chancengleichheit und Leistungsgerechtigkeit - neben dem erwähnten komplementären Verhältnis - ein Konflikt besteht.

\footnotetext{
Irene Becker, Dr. rer. pol., Dipl.-Volksw., arbeitet als freiberufliche Wissenschaftlerin in Riedstadt. Arbeitsschwerpunkte: Einkommens- und Vermögensverteilung, soziale Sicherung. e-mail: i-h.becker@t-online.de
} 
Teilweise bedingen sich also einzelne Ziele, sodass eine Maßnahme zur Annäherung an mehrere Ziele gleichzeitig führen kann. Teilweise begrenzen sie sich aber auch gegenseitig. (Politische) Kompromisse scheinen insbesondere zwischen Aspekten der Leistungsgerechtigkeit einerseits und den weiteren Zieldimensionen andererseits erforderlich $\mathrm{zu}$ sein. Allerdings steht dem auf den ersten Blick offenkundigen Konflikt zwischen Leistungs- und Bedarfsgerechtigkeit eine - über die Chancengerechtigkeit - mittelbare komplementäre Beziehung gegenüber. Zudem ist die implizite Annahme, die Markteinkommensverteilung entspräche dem Leistungsprinzip, kritisch zu hinterfragen. Wenn die untersten Arbeitseinkommen weniger die Leistung als vielmehr ein strukturelles Machtungleichgewicht und dadurch ermöglichtes Lohndumping spiegeln, würde eine stärkere Leistungsorientierung dieser Einkommen auch zu einer Annäherung an Bedarfsgerechtigkeit führen; in dieser Situation wären beide Teilziele komplementär. Analog wäre bei Verletzungen des Leistungsprinzips im obersten Segment der Einkommensverteilung infolge von Machtstrukturen und sozialen Schließungsprozessen sowie bei Bonuszahlungen an die Verantwortlichen von „Pleitefirmen“ eine stark progressive Besteuerung durchaus dem Teilziel der Leistungsgerechtigkeit förderlich. Letztlich hängt es also von der Ausgangssituation und schließlich auch von der empirischen Definition des Leistungsbegriffs $\mathrm{ab}$, ob und inwieweit eine gesetzliche Regelung bzw. politische Maßnahme mit einem Zielkonflikt verbunden ist. Vor dem Hintergrund dieser komplexen Zusammenhänge erscheinen Wertedebatten, in denen Teilziele als Alternativen behandelt werden, als verkürzt. Die häufige Reduzierung der sozialpolitischen Diskussion auf die angeblich eigenständigen Aspekte der Chancen- und der Verteilungs- oder Ergebnisgerechtigkeit verkennt wesentliche Bedingungskonstellationen, die auch einem dynamisch verstandenen Konzept der Verwirklichungschancen immanent sind. Der sogenannte „Dritte Weg“ bei der Gestaltung von Wohlfahrtsstaaten tendiert dazu, „aktivierende Maßnahmen als Ersatz herkömmlicher Politiken der Einkommenssicherung zu verstehen - wo doch die Linderung von Armut und Einkommensunsicherheit vielmehr eine notwendige Voraussetzung effektiver sozi-

Abb. 1: Soziale Gerechtigkeit - Teilziele und Zielinterdependenzen

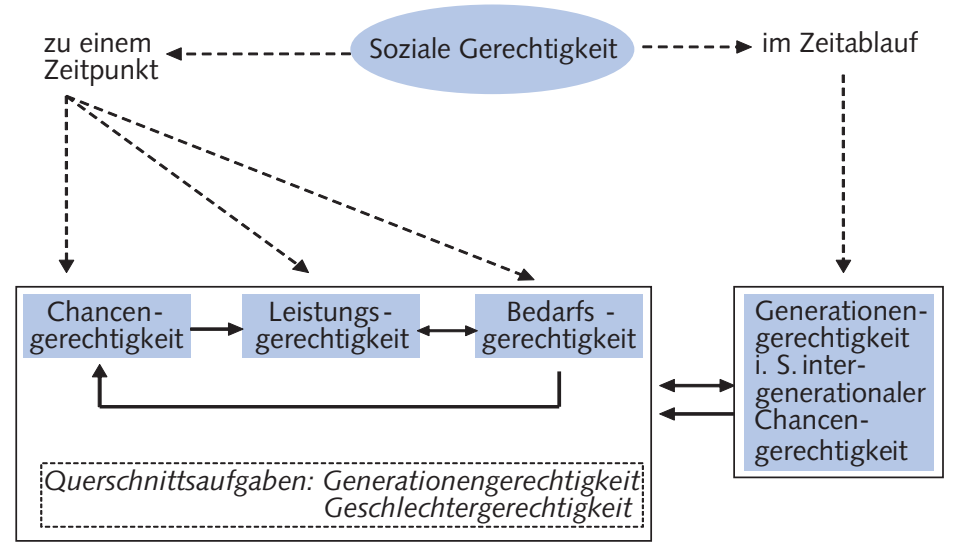

$\longleftrightarrow$ Konflikt

$\longrightarrow$ komplementäre Beziehung

Quelle: Darstellung der Autorin.

WSI MITTELUUNGEN

alinvestiver Strategien darstellt" (EspingAndersen 2004, S. 193).

Die weiteren Ausführungen beschränken sich auf die Einkommensverteilung im Sinne eines Spiegelbildes wesentlicher gesellschaftlicher Rahmenbedingungen und auf die Frage, inwieweit hier Zielannäherungen oder Entfernungen vom Ziel sozialer Gerechtigkeit erkennbar sind. ${ }^{1}$ Da die Teilziele sozialer Gerechtigkeit nicht verbindlich und allgemein gültig (qualitativ und quantitativ) abzugrenzen sind, kann es allerdings nicht um die Messung eines kardinal skalierten Zielerreichungsgrades, sondern nur um den „bescheidenen“ Ansatz der deskriptiven Annäherung an ein Bild über die Reichweite sozialer Gerechtigkeit in unserer Gesellschaft gehen. Die dabei herangezogenen Daten sind auf den Zeitraum bis 2006 beschränkt. Für 2007 und 2008 liegen bisher nur wenige Ergebnisse zum vorliegenden Kontext vor, die zudem methodisch nicht voll vergleichbar sind; sie deuten aber eine gleichbleibende Entwicklungsrichtung an. ${ }^{2}$

\section{Einkommensverteilung als ein Spiegelbild der Zielumsetzung}

\subsection{PROBLEME DER MESSUNG UND BEWERTUNG VON LEISTUNG}

Leistungsgerechtigkeit rekurriert auf eine ungleiche Ausgangslage und ungleiche
Leistungen von Individuen und damit auf ein Kriterium der relativen Gleichheit oder Gleichbehandlung (Döring 1994, S. 71). Letzteres äußert sich z. B. in der traditionellen gewerkschaftlichen Forderung „Gleicher Lohn für gleiche Arbeit!“, die den Umkehrschluss impliziert, dass absolute Einkommensunterschiede insoweit als gerecht anzusehen sind, als sie das Ergebnis unterschiedlicher Leistungen sind. Damit soll nicht nur individuelle Gerechtigkeit durch Gleichbehandlung, sondern auch ein Leistungsanreiz erreicht werden, der zu eigenen Anstrengungen führt und über die Erhöhung des Sozialprodukts für alle Gesellschaftsmitglieder von Vorteil ist.

Die Konkretisierung des Ziels der Leistungsgerechtigkeit ist allerdings strittig, da weder über die Abgrenzung noch über die individuelle Zurechnung und Bewertung von Leistung bzw. die Quantifizierung von Leistungsunterschieden Einvernehmen zu erreichen ist. Die einfachste und gängige Vorgehensweise besteht darin, „explizit oder implizit dem Markt die Definitionsmacht zu überlassen“" (Döring 1994, S. 72), wobei die Funktionsfähigkeit der Märkte unterstellt wird. Diese ist allerdings nicht selbstverständlich gegeben,

1 Ein breiteres Spektrum von Teilhabedimensionen wird in Becker/Hauser 2009 bearbeitet.

2 Vgl. z. B. Grabka/Frick 2010; die dort für das Jahr 2008 ausgewiesenen Ergebnisse basieren auf einer Befragung, die zwar 2008 durchgeführt worden ist, aber auf retrospektiv für 2007 erhobenen Einkommensdaten; es handelt sich also faktisch um Verteilungsergebnisse für 2007. 
sondern muss durch gestaltende Maßnahmen des Staates durchgesetzt werden, was nur teilweise gelingt. Insbesondere die Voraussetzung gleichgewichtiger Marktpartner ist nicht gegeben. Von daher und aus grundsätzlichen Erwägungen ist es in mehrfacher Hinsicht problematisch, die Güter- und Kapitalmärkten resultierende Verteilung der Erwerbs- und Vermögenseinkommen als leistungsgerecht anzuerkennen, denn

- viele Leistungen werden nicht über den Markt, sondern unentgeltlich erbracht, insbesondere von Frauen für die Familie (Versorgungs-, Erziehungs- und Pflegetätigkeiten);

- zudem ist die Funktionsfähigkeit des Marktes in Teilbereichen mehr oder minder eingeschränkt;

- schließlich orientiert sich die Entlohnung der Produktionsfaktoren überwiegend am Output, sodass sie letztlich durch die gesamtwirtschaftliche Nachfrage nach der jeweiligen Leistung bzw. deren „Knappheit" sowie die Marktmacht von Interessengruppen bestimmt wird. Individuelle Anstrengung und Einsatzbereitschaft werden also allenfalls mittelbar honoriert. keiten wird dennoch - mangels adäquater und quantifizierbarer Alternativen - meist das Markteinkommen als Indikator von Leistung herangezogen. aus dem Zusammenwirken von Arbeits-,

Trotz dieser grundlegenden Unzulänglich-

\subsection{LEISTUNGSGERECHTIGKEIT: DIE ROLLE VON GESELLSCHAFT UND POLITIK}

Um eine leistungsbezogene Entlohnung zu fördern, hat der Staat nur indirekte Möglichkeiten: Er kann Rahmenbedingungen für Vertragsgestaltungen setzen sowie das Marktergebnis durch eine progressive Besteuerung nach der Leistungsfähigkeit korrigieren. Bei den Rahmensetzungen für Vergütungssysteme ist in Deutschland aber eine große Zurückhaltung bzw. Rückführung zu beobachten. So hat die Finanzmarktkrise bisher nur eine vorsichtige und zögerliche Reaktion hervorgerufen mit dem Ziel, die Managergehälter stärker am mittelfristigen Unternehmenserfolg auszurichten. Zudem wurden mit den jüngsten Arbeitsmarktreformen Rahmensetzungen zurückgenommen, indem der Kündigungsschutz gelockert, die Möglichkeiten zur Befristung von Arbeitsverhältnissen erweitert und die Beschränkungen für Leiharbeit weitgehend aufgehoben wurden. Dies führte zu einer weiteren Schwächung der wegen des gesamtwirtschaftlich zu geringen Arbeitsplatzangebots ohnehin schwachen Verhandlungsposition der (meisten) Arbeitnehmerinnen und Arbeitnehmer, sodass die Gefahr besteht, dass vormals akzeptierte Grundsätze der Leistungsgerechtigkeit ausgehöhlt werden. Das Ziel leistungsgerechter Entlohnung wurde aber auch unmittelbar zurückgedrängt, indem - bei weiterhin fehlendem gesetzlichem Mindestlohn - seit der Hartz-IV-Reform nahezu jede Beschäftigung als zumutbar

\section{Tabelle 1: Durchschnittliche Bruttostundenlöhne/-gehälter der 41 - bis 55-jährigen Arbeitnehmer 1998 und $2006^{1}$ nach Geschlecht und beruflichem Bildungsabschluss in Relation (\%) zum jeweiligen Durch- schnittslohn der Gruppe ohne Berufsabschluss (= 100 \%)}

\begin{tabular}{|c|c|c|c|c|}
\hline & \multicolumn{2}{|c|}{ Männer² } & \multicolumn{2}{|c|}{ Frauen $^{3}$} \\
\hline & 1998 & 2006 & 1998 & 2006 \\
\hline Lehre & 103 & 109 & 105 & 114 \\
\hline Fachschule u. ä. ${ }^{4}$ & 113 & 123 & 122 & 121 \\
\hline Fachhochschule u. ä. ${ }^{5}$ & 148 & 169 & 128 & 146 \\
\hline Universität, TH & (167) & 174 & (184) & 184 \\
\hline \multicolumn{5}{|c|}{$\begin{array}{l}\text { Bei Fallzahlen von } 30 \text { bis unter } 100 \text { werden die Ergebnisse in Klammern gesetzt. } \\
{ }_{1} \text { ohne Auszubildende, ohne Personen in Werkstätten für Behinderte, in Altersteilzeit, auf einer ABM-/SAM-Stelle oder in } \\
\text { einer Arbeitsgelegenheit; ohne Personen mit gleichzeitigem Bezug von Lohnersatzleistungen im Befragungsmonat und ohne } \\
\text { Personen, für die sich ein Bruttostundenlohn unter } 2 € \text { ergibt. Bei der Berechnung der Bruttostundenlöhne wird von den } \\
\text { Monatseinkommen ohne Jahressonderzahlungen, } 4,35 \text { Wochen pro Monat und der angegebenen tatsächlichen Arbeitszeit } \\
\text { ausgegangen. Falls Überstunden in der Regel abgefeiert werden, wird bei der Ermittlung des Bruttostundenlohnes die tat- } \\
\text { sächliche Arbeitszeit abzüglich der Überstunden als Divisor eingesetzt. } \\
{ }^{2} \text { Referenzwerte der Männer ohne beruflichen Bildungsabschluss. } 13,15 €(1998) \text { bzw. } 14,05 €(2006) \text {. } \\
{ }^{3} \text { Referenzwerte der Frauen ohne beruflichen Bildungsabschluss. } 10,39 €(1998) \text { bzw. } 11,30 €(2006) \text {. } \\
{ }^{4} \text { Abschluss einer Berufsfachschule, Fachschule, Meisterausbildung, Beamtenausbildung o. ä. } \\
{ }^{5} \text { Abschluss einer Fachhochschule, Ingenieurschule, Fachschule oder Hochschule in der ehemaligen DDR, Hochschule im } \\
\text { Ausland. }\end{array}$} \\
\hline \multicolumn{3}{|c|}{$\begin{array}{l}\text { Quelle: SOEP 1998, 2006, Einkommen im Monat vor der Befragung; } \\
\text { Berechnungen der Autorin. }\end{array}$} & & EILUN \\
\hline
\end{tabular}

gilt und die neue Politik des „Forderns und Förderns" das Instrument der Arbeitsgelegenheit mit (lediglich) Mehraufwandsentschädigung erdacht hat. Der von diesen Maßnahmen ausgehende Lohndruck nach unten erfolgt unabhängig von Leistungsaspekten und losgelöst von dem Grundsatz, dass der Wert einer erbrachten - und somit nachgefragten - Vollzeittätigkeit nicht geringer angesetzt werden kann als die Reproduktionskosten zur Erhaltung dieser Arbeitskraft.

Doch auch abgesehen von diesen Folgen der Deregulierung der Arbeitsmärkte stellt sich die allgemeine Frage, ob sich Leistung in Deutschland „lohnt“. Tabelle 1 gibt hierzu eine erste Teilantwort. Sie stellt die Veränderungen sogenannter Ausbildungsrenditen beispielhaft für die Altersgruppe der 41- bis 55-Jährigen und ohne Berücksichtigung der Selbstständigen dar. Die ausgewiesenen Prozentsätze kennzeichnen die nach dem beruflichen Abschluss differenzierten Durchschnittslohnsätze als Vielfaches des Durchschnittslohns der Männer bzw. Frauen ohne beruflichen Abschluss mittleren Alters. So zeigt sich beispielsweise, dass eine Lehre im Durchschnitt zu einem nur wenig über dem Referenzwert liegenden Stundenlohn führt, während ein Universitätsabschluss eine durchschnittliche Rendite von etwa $70 \%$ hat. Zwischen 1998 und 2006 haben sich die gruppenspezifischen Vergütungsniveaus tendenziell auseinanderentwickelt - zumindest die Investition in eine Ausbildung lohnt sich also nach wie vor, und zwar eher zu- als abnehmend. ${ }^{3}$

Hinter der Veränderung der Durchschnittslöhne-auch bei ausbildungs- und geschlechtsspezifischer Differenzierung - verbirgt sich eine generell zunehmende Spreizung der Arbeitnehmerentgelte. Tabelle 2 beschränkt sich auf den unteren und oberen Verteilungsrand und zeigt die relativen Häufigkeiten in den Jahren 1998, 2003 und 2006. Weniger als zwei Drittel des Medianlohnes - das sind etwa $9 €$ haben 1998 knapp 16 \% der unselbstständig Beschäftigten erhalten, 2006 waren es ca. $21 \%$. Dabei sind Frauen von Niedriglöhnen besonders betroffen. Aber auch der obere Bereich hat sich ausgeweitet, sodass der Bereich mittlerer Lohnsätze im Beobachtungszeitraum geschrumpft ist. Wenn

\footnotetext{
3 Vgl. dazu ausführlich Becker/Hauser 2009,
} S. $172-175$, S. 193-197. 
sich Leistung tendenziell weniger lohnt, dann ist es also eher am unteren als am oberen Rand.

Die anwachsenden oberen Entgeltgruppen - bzw. hohe Einkommensschichten generell-haben im Beobachtungszeitraum zudem in besonderem Maße von Einkommensteuerentlastungen profitiert. Denn die Steuerreformen seit 2000 haben zu einer deutlichen Senkung des Spitzensteuersatzes geführt - von $51 \%$ im Jahr 2000 auf derzeit $42 \%$ bzw. $45 \%$ oberhalb eines zu versteuernden Einkommens von $250.730 €(501.460 €$ beiEhepaaren)-, und die jüngsten Konjunkturpakete nochmals zu einer Abschwächung der Progression. Damit werden Abweichungen der Marktergebnisse von leistungsorientierten Vergütungen sowie Ergebnisse des Leistungsprinzips tendenziell weniger korrigiert. Die Einkommenseffekte der Tarifentlastungen zwischen 1998 und 2004 fielen trotz des deutlich reduzierten Eingangssteuersatzes umso stärker aus, je höher das zu versteuernde Einkommen ist (Corneo 2003). Ab einem zu versteuernden Einkommen von einer halben Million Euro resultierte eine Nettoeinkommenssteigerung um etwa ein Fünftel gegenüber nur etwa $6 \%$ bei Einkommen bis zu $50.000 €$, wobei die letzte Stufe der Steuerreform 2000 noch nicht berücksichtigt ist (ebd., S. 3); die Unterschiede zwischen den absoluten Einkommenssteigerungen sind noch drastischer.

Die jüngsten Änderungen des Einkommensteuertarifs werden teilweise mit Verweis auf die sogenannte „kalte Progression" gerechtfertigt: Bei inflationären Entwicklungen werden die zu versteuernden Einkommen zunehmend von der Steuerprogression erfasst, mit der Folge, dass die Belastung eines gegebenen Realeinkommens im Zeitablauf steigt. Um dem entgegenzuwirken, wäre es aber angemessener, die obere Proportionalzone nach oben zu verschieben und die Progression im darunter liegenden Bereich zu mildern, ohne den Spitzensteuersatz zu senken.

Die zunehmende Spreizung der Erwerbseinkommen bei anhaltend hoher Arbeitslosigkeit sowie eine hohe oder sogar tendenziell zunehmende Ungleichheit der Vermögensverteilung (Frick et al. 2010) mit der Folge entsprechend ungleich verteilter Kapitaleinkommen spiegeln sich in einer weiteren Spreizung der Markteinkommen. Wenn ein um die Einkommen aus staatlichen Alterssicherungssystemen erweiterter Markteinkommensbegriff zugrundegelegt

Tabelle 2: Relative Häufigkeiten der Randbereiche der Stundenlohnverteilung ${ }^{1}$ - in \% -

\begin{tabular}{|c|c|c|c|}
\hline & 1998 & 2003 & 2006 \\
\hline \multicolumn{4}{|c|}{ Bruttostundenlohn $<2 / 3$ des Median } \\
\hline Frauen & 22,0 & 26,7 & 27,1 \\
\hline Männer & 10,9 & 12,4 & 14,6 \\
\hline \multirow[t]{2}{*}{ insgesamt } & 15,7 & 19,2 & 20,7 \\
\hline & \multicolumn{3}{|c|}{ Bruttostundenlohn >= doppelter Median } \\
\hline Frauen & 2,5 & 3,0 & 4,2 \\
\hline Männer & 6,7 & 8,5 & 8,8 \\
\hline insgesamt & 4,9 & 5,9 & 6,5 \\
\hline \multicolumn{4}{|c|}{${ }^{1}$ Zur Abgrenzung der Gesamtgruppe vgl. Fußnote 1 zu Tabelle 1.} \\
\hline \multicolumn{3}{|c|}{$\begin{array}{l}\text { Quelle: SOEP 1998, } 2003 \text { und 2006, Einkommen im Monat vor der Befragung; } \\
\text { Berechnungen der Autorin. }\end{array}$} & \\
\hline
\end{tabular}

und eine Äquivalenzgewichtung gemäß der modifizierten OECD-Skala vorgenommen wird (erweitertes Marktäquivalenzeinkommen), zeigt sich ein Anstieg des Gini-Koeffizienten als zusammenfassendem Ungleichheitsmaß von 0,347 im Jahr 2000 um $13 \%$ auf 0,393 im Jahr 2006. Angesichts dieser Entwicklung, der Veränderungen des Einkommensteuertarifs und restriktiver Tendenzen in der Sozialpolitik ist es letztlich nicht verwunderlich, dass auch die Ungleichheit der bedarfsgewichteten Nettoeinkommen zugenommen hat. So ist der Gini-Koeffizient der Nettoäquivalenzeinkommen (Bezug: modifizierte OECD-Skala) um fast $16 \%$ von 0,261 (2000) auf 0,302 (2006) gestiegen. Der ausgleichende Effekt des Abgabenund Transfersystems war also rückläufig. Dies spiegelt sich anschaulich in der Verteilung des Nettoäquivalenzeinkommens nach Bevölkerungsdezilen. Die unteren $10 \%$ erreichten im Jahr 2006 mit 3,4 \% des Einkommensaggregats etwa ein Zehntel weniger als 2000, während auf das obere Zehntel im Jahr 2006 fast ein Viertel des Gesamten entfällt - das entspricht einem Zuwachs um gut $13 \%$ (Becker 2011). Eine allgemein zu geringe oder gar abnehmende Einkommensdifferenzierung, die leistungshemmend sein könnte, ist jedenfalls nicht erkennbar.

\subsection{ASPEKTE DER BEDARFS- GERECHTIGKEIT}

Der grundgesetzlich vorgeschriebene Schutz der Menschenwürde setzt neben der Bereitstellung von öffentlichen und meritorischen ${ }^{4}$ Gütern - z. B. Verteidigung, innere Sicherheit, Infrastruktur auf der individuellen Ebene zumindest die Deckung des soziokulturellen Existenzminimums voraus, und zwar unabhängig von produktiver Erwerbstätigkeit oder konformen Verhaltensweisen. Dies hat das Bundesverfassungsgericht in seinem sogenannten „Hartz-IV-Urteil“" vom 9. Februar 2010 zur Bemessung der Regelleistungen der Grundsicherung nach dem Zweiten Buch bzw. Zwölften Buch Sozialgesetzbuch (SGB II bzw. XII) eindrucksvoll bestätigt (BVerfG, 1 BvL 1/09). Damit sind dem Leistungsprinzip Grenzen gesetzt. Da die Achtung der Menschenwürde ein Verbot systematischer Ausgrenzungen von Gesellschaftsmitgliedern impliziert, ist nicht nur das physische Überleben zu sichern, sondern ein Lebensstandardniveau in Relation zu den gesamtgesellschaftlichen Verhältnissen und unter Einbeziehung von sozialen und kulturellen Bedürfnissen. Die Abgrenzung gesellschaftlich anerkannter Mindestbedarfe von grenzenlosen Bedürfnissen und die Frage, wie weitgehend Unterschiede der Bedarfsdeckungsmöglichkeiten einzelner Gruppen durch staatliche Maßnahmen anzugleichen sind, wird allerdings äußerst kontrovers diskutiert und muss letztlich im demokratischen Prozess entschieden bzw. im gesellschaftlichen Wandel immer wieder revidiert werden.

Die Politik der vergangenen Jahre hat dem Ziel der Bedarfsgerechtigkeit keinen besonderen Stellenwert eingeräumt. Dies zeigt sich an folgenden Beispielen:

- Das Kindergeld wurde nach 2002 erstmals 2009 erhöht und bleibt auch nach der weiteren Erhöhung zum Januar 2010

\footnotetext{
4 Meritorische Güter unterscheiden sich von privaten Gütern dadurch, dass sich der Konsumnutzen nicht auf die Konsumenten beschränkt: Infolge von externen Effekten steigt auch der Nutzen der Gesamtgesellschaft; beispielsweise wird durch Gesundheit und Bildung nicht nur die individuelle Wohlfahrt, sondern auch das gesamtwirtschaftliche Potenzial erhöht.
} 
Tabelle 3: Quoten relativer Einkommensarmut - in \% -

\begin{tabular}{|c|c|c|c|c|c|c|}
\hline & \multirow[t]{2}{*}{ Gesamtbevölkerung } & \multicolumn{5}{|c|}{ Alter von ... bis ... Jahre } \\
\hline & & $0-15$ & $16-24$ & $25-49$ & $50-64$ & 65 u. m. \\
\hline 1999 & 10,7 & 13,9 & 15,6 & 8,7 & 9,1 & 11,1 \\
\hline 2006 & 14,9 & 19,8 & 23,8 & 14,0 & 10,7 & 12,3 \\
\hline
\end{tabular}

hinter dem kindlichen Existenzminimum zurück. Der 2005 eingeführte und 2008 reformierte Kinderzuschlag hat infolge seiner restriktiven und komplizierten Ausgestaltung bisher nur geringe Wirkung gezeigt.

- Die Ersetzung des Erziehungs- durch das Elterngeld impliziert eine Verkürzung der maximalen Bezugsdauer um zehn Monate, was für viele Familien gerade am unteren Rand der Einkommensverteilung problematisch ist. Zudem wird das Elterngeld ab Januar 2011 vollständig auf Arbeitslosengeld-(ALG)II-Ansprüche angerechnet, was einer Einkommensminderung für leistungsberechtigte junge Eltern um $300 €$ pro Monat entspricht.

- Die Wohngeldnovelle zum Januar 2009 brachte immerhin Verbesserungen, allerdings nach Zeiten der "Aushöhlung“ durch eine fehlende Dynamisierung. Die erstmals eingeführte Heizkostenpauschale für Wohngeldempfänger wurde ab Januar 2011 wieder zurückgenommen.

- Die Reformen der vormals universellen Sozialhilfe brachten für die alte Generation Verbesserungen (Grundsicherung im Alter und bei Erwerbsminderung; SGB XII), für Langzeitarbeitslose aber wegen der Abschaffung der Arbeitslosenhilfe und vieler Detailregelungen (Grundsicherung für Arbeitsuchende; SGB II) eher Verschlechterungen.

- Und schließlich hat sich das derzeitige Verfahren zur Bemessung des Grundsicherungsniveaus als unzulänglich erwiesen, wie mehrere Expertisen belegt haben und vom Bundesverfassungsgericht in seinem Urteil vom 9. Februar 2010 festgestellt wurde. Auch die gerade beschlossene Neuregelung zum Januar 2011 wird von mehreren Wissenschaftlerinnen und Wissenschaftlern sowie Verbänden wegen ihres zu geringen Niveaus kritisiert und als nicht verfassungsgerecht eingestuft.

Wie sich diese Politik im Zusammenhang mit den gesamtwirtschaftlichen Rahmenbedingungen ausgewirkt hat, zeigt bei- spielsweise die Entwicklung von Quoten relativer Einkommensarmut. Die in Tabelle 3 ausgewiesenen Ergebnisse beziehen sich auf die EU-weit vereinbarte „Armutsrisikoschwelle“von $60 \%$ des Median der Nettoäquivalenzeinkommen, wobei die modifizierte OECD-Skala zugrunde zu legen ist. Von 2000 bis 2006 ist die Armutsquote um etwa $40 \%$ - von $10,7 \%$ auf $14,9 \%$ - gestiegen (Becker 2011). Nach Berechnungen des Deutschen Instituts für Wirtschaftsforschung (DIW) in Berlin hat die Quote von 2006 auf 2007 weiter zugenommen (Grabka/Frick 2010, S. 5) - der Trend scheint also ungebrochen zu sein. Besonders betroffen von dieser Entwicklung sind Kinder und Jugendliche sowie deren „Elterngeneration“, die 25- bis 49-Jährigen. Dies ist nicht nur auf die bis 2005 gestiegene Arbeitslosenquote $(13 \%)^{5}$ und die besonders hohe Armutsquote der Haushalte mit überwiegendem Einkommen aus Arbeitslosengeld oder Arbeitslosenhilfe bzw. ALG II (2001: $66 \%$; 2006: 70 \%) zurückzuführen. Vielmehr ist auch die Armutsquote der Haushalte mit überwiegendem Einkommen aus Erwerbstätigkeit gestiegen. ${ }^{6}$

Die Entwicklung des Armutsproblems ist umso gravierender, als bei einem zunehmenden Anteil der Betroffenen Einkommensarmut zu einem Dauerzustand wird. Im Jahr 2000 lebten $59 \%$ der Armutsbevölkerung bereits in zwei von drei Vorjahren unterhalb der relativen Armutsgrenze, im Jahr 2005 waren es schon 66 \% (DIW et al. 2008, S. 104).

\subsection{ASPEKTE DER CHANCEN- GERECHTIGKEIT}

Das Ziel der Gleichheit der Chancen für eine selbstbestimmte Lebensgestaltung wird meist vorrangig auf die Startchancen bezogen, wobei der Start ins Leben - also die Chancen zum Zeitpunkt der Geburt-, der Start in das Bildungs- und Ausbildungssystem oder der berufliche Start betrachtet werden kann. Darüber hinaus ergeben sich Chancenunterschiede teilweise aber erst im weiteren Lebensverlauf-beispielsweise nach einem Unfall oder einer Erkrankung mit der Folge einer Behinderung oder infolge von wirtschaftlichem Strukturwandel, der zur Entwertung oder aber Aufwertung individueller Fähigkeiten und Bildungsabschlüsse führen kann. Die Chancen zu den verschiedenen Zeitpunkten im Lebensverlauf sind letztlich von einer Vielzahl von Faktoren und Konstellationen abhängig und dementsprechend ungleich verteilt. Neben unterschiedlichen Veranlagungen und Begabungen, unterschiedlichen Förderungen durch das Elternhaus und verschiedenartigen Diskriminierungen sind geschlechtsspezifische Rollenverteilungen, die Arbeitsmarktsituation und generell die historischen bzw. gesamtgesellschaftlichen Rahmenbedingungen in einzelnen Lebensabschnitten und nicht zuletzt Vermögensübertragungen von wesentlicher Bedeutung. Von staatlicher Seite kann auf mehreren Ebenen korrigierend eingegriffen werden.

Ein Rückblick auf die institutionellen Rahmensetzungen zur Annäherung an Chancen- bzw. formale Zugangsgerechtigkeit ergibt ein zwiespältiges Bild (vgl. hierzu auch Hopf in diesem Heft). Zum einen sind in jüngster Zeit richtungweisende Gesetze erlassen worden, insbesondere um die Möglichkeiten der außerhäuslichen Kinderbetreuung zu verbessern. Damit werden Erfordernisse der Chancengerechtigkeit sowohl für Kinder - beim Zugang zu Bildungsinstitutionen - als auch für Frauen - beim Zugang zum Arbeitsmarkt - angegangen. Die Umsetzung der gesetzgeberischen Anstöße erfolgt allerdings nur allmählich, steht faktisch unter Finanzierungsvorbehalten bzw. geht teilweise zulasten der Betreuungsqualität und kommt für viele Menschen zu spät. Neben den Investitionen in die Betreuungs-Infrastruktur wurden mit der Einführung des Elterngeldes auch bei den monetären Transfers neue Schwerpunkte gesetzt. Da die maximale Bezugsdauer an eine Erwerbsunterbrechung auch des Vaters geknüpft ist, wurde ein Anstoß gegeben, um bestehende Rollenmuster aufzubrechen. Zum anderen haben sich aber durch die Aushöhlung

5 Anteil der Arbeitslosen an den abhängigen Erwerbspersonen (ohne Soldaten).

6 Die Armutsquote der Haushalte von Arbeitnehmern (Selbstständigen) lag 2001 bei 8,3\% (3,6\%), 2006 bei 10,0\% (5,2\%), vgl. Becker 2011. 
der Lehr- und Lernmittelfreiheit und die Einführung von Studiengebühren neue Hürden im Bildungssystem aufgebaut, welche die Teilhabe unterer Einkommensschichten erschweren oder verhindern und damit dem Ziel der Chancengerechtigkeit entgegenstehen. Insgesamt hält das öffentliche Engagement im Bildungssektor mit den proklamierten Bildungszielen nicht Schritt. Dies zeigt sich an dem bei knapp $5 \%$ stagnierenden Anteil der Bildungsausgaben am Bruttoinlandsprodukt und an ungefähr konstanten Schüler-Lehrer-Relationen an allgemein bildenden Schulen (Becker/Hauser 2009, S. 68f.). Schließlich wird weiterhin auf eine Korrektur der Vermögensverteilung weitgehend verzichtet. Die jüngste Erbschaftsteuerreform hat vielfältige Entlastungen und nur für Erbschaftsfälle bei entfernten Verwandten eine Anhebung der Steuersätze gebracht - hier werden die Konsequenzen für das Ziel der Chancengerechtigkeit also ausgeblendet.

Ob die Ansätze zur Verbesserung frühkindlicher Bildung und die Reformen der schulischen Bildung zu einer merklichen Angleichung der Chancen während der Ausbildungsphase $\mathrm{e}^{7}$ und darüber hinaus auch im Erwerbsleben führen werden, bleibt abzuwarten. Für mittelfristige Erfolge sind die Arbeitsmarktentwicklung und damit die Arbeitsmarkt- und Wirtschaftspolitik mitentscheidend. Bisher sind die Arbeitsmarktchancen auch infolge makroökonomischer Zusammenhänge sehr ungleich verteilt. Über Jahre ist - bei Rückführung von Arbeitsbeschaffungsund Strukturanpassungsmaßnahmen keine Stabilisierung der Binnennachfrage durch Staatsausgaben erfolgt; erst mit der weltweiten Wirtschaftskrise ist mit „Konjunkturpaketen“" auch die Staatsnachfrage ausgeweitet worden. Dennoch und trotz der mit dem Instrument des Kurzarbeitergeldes abgefederten Folgen der Finanzmarktkrise sind die Beschäftigungsprobleme nicht gelöst. Dass Bildungserfolge nicht „automatisch“ "zu entsprechenden Erfolgen im Erwerbsleben führen, hat sich bei den Frauen gezeigt; obwohl sie hinsichtlich schulischer und beruflicher Bildungserfolge aufgeholt bzw. die Männer teilweise überholt haben, hinken sie bei Karrierechancen und Entgeltpositionen weiterhin hinterher (Becker/Hauser 2009, S. 163ff.).

Vorliegende empirische Indikatoren zur Bildungsbeteiligung und zur sozialen Mobilität zeichnen ein ernüchterndes Bild. Im oberen Teil der Tabelle 4 ist die Beteili-

\begin{tabular}{|c|c|c|c|c|c|}
\hline \multicolumn{6}{|c|}{$\begin{array}{l}\text { Tabelle 4: Beteiligung auf weiterführenden Ebenen des Bildungssys- } \\
\text { tems nach sozialer Herkunft, } 1996 \text { und } 2005 \text { - Beteiligungsquoten in \% - }\end{array}$} \\
\hline \multicolumn{6}{|c|}{ Schulbildung des Vaters ${ }^{1}$} \\
\hline & Hauptschule & Realschule & FH-Reife & allg. HS-Reife & insgesamt \\
\hline \multicolumn{6}{|c|}{ Beteiligung an Sekundarstufe II (Klassen 11 bis 13, gymnasiale Oberstufe) $)^{2}$} \\
\hline 1996 & 33 & 47 & 73 & 84 & 48 \\
\hline 2005 & 36 & 49 & 78 & 83 & 54 \\
\hline \multicolumn{6}{|c|}{ Beteiligung an Hochschulen (Studienanfängerquoten) ${ }^{3}$} \\
\hline 1996 & 18 & 29 & & & 27 \\
\hline 2005 & 19 & 26 & & & 37 \\
\hline \multicolumn{6}{|c|}{$\begin{array}{l}1 \text { Bei Kindern von alleinerziehenden Frauen gilt die Schulbildung bzw. berufliche Stellung der Mutter als soziale Herkunftsvariable. } \\
2 \text { Schüler/Schülerinnen der Klassenstufen } 11 \text { bis } 13 \text { in Relation zu allen } 17 \text { - bis } 18 \text {-Jährigen der jeweiligen Herkunftsgruppe. } \\
3 \text { S Studienanfänger / -anfängerinnen im ersten Hochschulsemester an allen } 19 \text { - bis } 24 \text {-Jährigen der jeweiligen Herkunftsgruppe. }\end{array}$} \\
\hline $\begin{array}{l}\text { Quelle: } \\
\text { amtlich } \\
\text { (z. B. H }\end{array}$ & $\begin{array}{l}\text { tedt et al. 2007, S } \\
\text { stik (z. B. des Mikr } \\
\text { dienanfängerbefra }\end{array}$ & $\begin{array}{l}\text { 6, } 98,102,103 \\
\text { us) und andere } \\
\text { n). }\end{array}$ & $\begin{array}{l}\text { sis: } \\
\text { nungsreihen }\end{array}$ & W & ITTEILUNGEN \\
\hline
\end{tabular}

gung an der Sekundarstufe II differenziert nach dem Schulabschluss des Vaters bzw. bei Alleinerziehenden der Mutter ausgewiesen. Im Jahr 2005 besuchte nur gut ein Drittel der 17- bis 18-Jährigen, deren Vater bzw. Mutter lediglich einen Hauptschulabschluss hat, eine weiterführende Schule - gegenüber 1996 hat sich kaum etwas verändert. Von denjenigen dieser Altersgruppe, deren Vater bzw. Mutter die allgemeine Hochschulreife erworben hat, sind demgegenüber mehr als vier Fünftel auf einer Schule der Sekundarstufe II. ${ }^{8}$ Noch gravierender sind die Unterschiede bei den Studienanfängerquoten, die im unteren Teil der Tabelle ausgewiesen sind.

Der Bildungsweg von Kindern und Jugendlichen ist also weiterhin sehr stark vom formalen Bildungsabschluss, aber auch von der beruflichen Stellung und der Einkommenssituation der Eltern (Becker/ Hauser 2009, S. 157, 159; vgl. hierzu auch Hopf in diesem Heft) abhängig. Die genannten Einflussgrößen korrelieren zwar erheblich; nach neuen Forschungsergebnissen ist aber „Armut nicht nur ein weiterer Indikator für die soziale Herkunft von Schülerinnen und Schülern, sondern liefert bei gleichzeitiger Berücksichtigung der Bildungsnähe und der beruflichen Stellung zusätzliche Erkenntnisse zum Bildungserfolg von Kindern und Jugendlichen. Insbesondere in bildungsnahen Familien ist die Leistungsdifferenz zwischen armutsgefährdeten und nicht armutsgefährdeten Kindern relativ groß" (Bos et al. 2010, S. 70). Dementsprechend sind seit Mitte der 1970er Jahre eher mäßige Veränderungen der sozialen Mobilität zu beobachten; bei den Frauen in Westdeutschland haben die Aufwärts- und Abwärtsmobilitätsraten allerdings erheblich zugenommen, sodass eine Annäherung an die Mobilitätsraten der Männer erfolgt ist (Pollak 2008,
2010). Besonders problematisch ist jedoch die noch immer hohe Immobilität bei den Gruppen aus „bildungsfernen“ Familien. Im Beobachtungszeitraum 2000 bis 2006 arbeitete gut ein Viertel der Männer, deren Vater ungelernter Arbeiter war, wiederum als Ungelernter, bei den Frauen waren es sogar fast zwei Fünftel. ${ }^{9}$

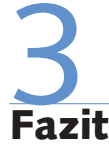

Auch wenn die Bedeutung der formalen Zugangsgerechtigkeit für das Ziel sozialer Gerechtigkeit unbestritten ist, so verbleiben doch offensichtlich systemische Chancenunterschiede, deren Effekte auf die Verteilungsergebnisse wiederum die Chancen der Folgeperiode bzw. der nächsten Generation beeinflussen. Wenn von unterschiedlichen Begabungen und Präferenzen abgesehen wird, sind die individuellen Teilhabeergebnisse und damit auch das Einkommen und die damit verbundenen Konsum- und Sparvorgänge entscheidend von den Ressourcen zum Ausgangszeitpunkt sowie von der Kombination gesellschaftlicher und individueller Umwandlungsfaktoren in einzelnen Lebensphasen abhängig. Und sie bestimmen wiederum über Rückkoppelungseffekte auf Ressourcen und individuelle Umwandlungsfaktoren maßgeblich

$7 \quad$ Immerhin gibt es erste Anzeichen, dass der in Deutschland sehr starke Zusammenhang zwischen sozialer Herkunft und Schülerleistung sich dem OECD-Durchschnitt nähert; vgl. Becker/ Hauser 2009, S. $154 f$.

8 Zur Differenzierung nach alten und neuen Bundesländern vgl. Merten 2010, S. 155.

9 Eine ausführlichere Darstellung der Ergebnisse von Pollak (2008) findet sich in Becker/Hauser 2009 S. $160 \mathrm{ff}$. 
die Verwirklichungschancen für sich selbst und für die Kinder in der Folgeperiode.

Vor diesem Hintergrund wäre es verkürzt, staatliche Verantwortung allein oder vorrangig auf die gesellschaftlichen Umwandlungsfaktoren bzw. auf den Teil, der für staatlich beeinflussbar gehalten wird, $\mathrm{zu}$ beziehen und von monetären Transfers abzusehen. Dies gilt insbesondere in Anbetracht der aufgezeigten lang- fristig prägenden Sozialstruktur: Wenn die Gewährleistung akzeptabler Verwirklichungschancen durch gesellschaftliche Umwandlungsfaktoren - insbesondere durch Bildungs- und Betreuungsangebote - nicht oder nur teilweise gelingt bzw. bisher gelungen ist, sind die Teilziele der Bedarfsgerechtigkeit und der Chancengerechtigkeit nicht voneinander zu trennen. Dementsprechend ist eine „periodische“
Umverteilung über Transfers erforderlich (vgl. hierzu auch Kronauer/Schmid in diesem Heft), deren notwendiges Ausmaß freilich auch vom Umfang und Erfolg infrastruktureller Maßnahmen zur Umsetzung von Chancengerechtigkeit abhängig ist. Nur unter diesen Voraussetzungen werden auf Autonomie zielende sozialinvestive Strategien von Erfolg gekrönt sein.

\section{LITERATUR}

Bartelheimer, P. (2007): Politik der Teilhabe. Ein soziologischer Beipackzettel, Fachforum: Analysen und Kommentare 1, hrsg. von der Friedrich-Ebert-Stiftung, Berlin

Becker, I. (2011): Einkommen und Einkommensverteilung, in: Forschungsverbund Sozioökonomische Berichterstattung (Hrsg.): Zweiter Bericht zur sozioökonomischen Entwicklung Deutschlands, Wiesbaden (im Erscheinen)

Becker, I./Hauser, R. (2009): Soziale Gerechtigkeit - ein magisches Viereck. Zieldimensionen, Politikanalysen und empirische Befunde, Berlin

Bos, W./Stubbe, T./Buddeberg, M. (2010): Einkommensarmut und schulische Kompetenzen, in: Fischer, J./Merten, R. (Hrsg.): Armut und soziale Ausgrenzung von Kindern und Jugendlichen. Problembestimmung und Interventionsansätze, Baltmannsweiler, S. 58-72

Corneo, G. (2003): Verteilungsarithmetik der rot-grünen Einkommenssteuerreform, Diskussionsbeitrag 2003/14, Fachbereich Wirtschaftswissenschaften, Universität Osnabrück

Deutsches Institut für Wirtschaftsforschung (DIW) (Grabka, M.)/ Zentrum für Europäische Wirtschaftsforschung (ZEW) (Westerheide, P.)/Hauser, R./Becker, I. (2008): Integrierte Analyse der Einkommensund Vermögensverteilung. Abschlussbericht zur Studie im Auftrag des Bundesministeriums für Arbeit und Soziales, Reihe Lebenslagen in Deutschland, Bonn

Döring, D. (1994): Anmerkungen zum Gerechtigkeitsbegriff der Sozialpolitik mit besonderer Berücksichtigung liberaler Vorstellungen zur Sozialversicherungspolitik, in: Döring, D./Nullmeier, F./Pioch, R./Vobruba, G. (Hrsg.): Gerechtigkeit im Wohlfahrtsstaat, Reihe "Standortdebatte“, hrsg. von der der Hans-Böckler-Stiftung, Marburg, S. 67-113

Esping-Andersen, G. (2003): Herkunft und Lebenschancen. Warum wir eine neue Politik gegen soziale Vererbung brauchen, in: Berliner Republik (6); http://www.b-republik.de/archiv/herkunft-und-lebenschancen

Esping-Andersen, G. (2004): Die gute Gesellschaft und der neue Wohlfahrtsstaat, in: Zeitschrift für Sozialreform 50 (1-2), Schwerpunkt: Eine neue Architektur der Sozialen Sicherung in Deutschland?, hrsg. von A. Evers, S. 189-210
Fischer, J./Merten, R. (Hrsg.) (2010): Armut und soziale Ausgrenzung von Kindern und Jugendlichen. Problembestimmung und Interventionsansätze, Baltmannsweiler

Frick, J. R./Grabka, M. M./Hauser, R. (2010): Die Verteilung der Vermögen in Deutschland. Empirische Analysen für Personen und Haushalte, Berlin

Grabka, M. M./Frick, J. R. (2010): Weiterhin hohes Armutsrisiko in Deutschland: Kinder und junge Erwachsene sind besonders betroffen, in: Wochenbericht des DIW Berlin (7), S. 2-11

Isserstedt, W./Middendorff, E./Fabian, G./Wolter, A. (2007): Die wirtschaftliche und soziale Lage der Studierenden in der Bundesrepublik Deutschland 2006. 18. Sozialerhebung des Deutschen Studentenwerks durchgeführt durch HIS Hochschul-InformationsSystem, hrsg. vom Bundesministerium für Bildung und Forschung (BMBF), Bonn/Berlin

Merten, R. (2010): Jugend und Armut - Herausforderungen angesichts einer vergessenen Generation, in: Fischer, J./Merten, R. (Hrsg.): Armut und soziale Ausgrenzung von Kindern und Jugendlichen. Problembestimmung und Interventionsansätze, Baltmannsweiler, S. 131-159 Pollak, R. (2008): Soziale Mobilität, in: Statistisches Bundesamt (Destatis)/Gesellschaft Sozialwissenschaftlicher Infrastruktureinrichtungen (GESIS-ZUMA)/Wissenschaftszentrum Berlin für Sozialforschung (WZB) (Hrsg.): Datenreport 2008, Zahlen und Fakten über die Bundesrepublik Deutschland, S. 180-187

Pollak, R. (2010): Kaum Bewegung, viel Ungleichheit. Eine Studie zu sozialem Auf- und Abstieg in Deutschland, Band 5 der Reihe Wirtschaft und Soziales, herausgegeben von der Heinrich-Böll-Stiftung, Berlin Sen, A. (1999): Commodities and Capabilities, second impression (first published in India 1987), Oxford

Zacher, H. F. (1977): Sozialstaatsprinzip, in: Handwörterbuch der Wirtschaftswissenschaft (HdWW), Band 7, Stuttgart/New York/Tübingen/ Göttingen/Zürich, S 152-160 